Article

\title{
The Exponentiated Truncated Inverse Weibull-Generated Family of Distributions with Applications
}

\author{
Abdullah M. Almarashi ${ }^{1}$, Mohammed Elgarhy ${ }^{2}$, Farrukh Jamal ${ }^{3} \mathbb{D}$ and Christophe Chesneau ${ }^{4, *}$ \\ 1 Statistics Department, Faculty of Science, King AbdulAziz University, Jeddah 21551, Saudi Arabia; \\ almarashi.research@gmail.com \\ 2 Valley High Institute for Management Finance and Information Systems, Obour, Qaliubia 11828, Egypt; \\ m_elgarhy85@sva.edu.eg \\ 3 Department of Statistics, Govt. S.A Postgraduate College Dera Nawab Sahib, Bahawalpur, \\ Punjab 63100, Pakistan; drfarrukh1982@gmail.com \\ 4 Department of Mathematics, Université de Caen, LMNO, Campus II, Science 3, 14032 Caen, France \\ * Correspondence: christophe.chesneau@gmail.com; Tel.: +33-02-3156-7424
}

Received: 16 March 2020; Accepted: 13 April 2020; Published: 20 April 2020

\begin{abstract}
In this paper, we propose a generalization of the so-called truncated inverse Weibull-generated family of distributions by the use of the power transform, adding a new shape parameter. We motivate this generalization by presenting theoretical and practical gains, both consequences of new flexible symmetric/asymmetric properties in a wide sense. Our main mathematical results are about stochastic ordering, uni/multimodality analysis, series expansions of crucial probability functions, probability weighted moments, raw and central moments, order statistics, and the maximum likelihood method. The special member of the family defined with the inverse Weibull distribution as baseline is highlighted. It constitutes a new four-parameter lifetime distribution which brightensby the multitude of different shapes of the corresponding probability density and hazard rate functions. Then, we use it for modelling purposes. In particular, a complete numerical study is performed, showing the efficiency of the corresponding maximum likelihood estimates by simulation work, and fitting three practical data sets, with fair comparison to six notable models of the literature.
\end{abstract}

Keywords: truncated family of distributions; inverse Weibull distribution; moments; order statistics; maximum likelihood method; simulation; data analysis

MSC: 60E05; 62E15; 62F10

\section{Introduction}

As an established statistical fact, a lot of current data sets cannot be properly fitted by the existing distributions. For this reason, new families of continuous distributions are developed with the aim to offer (continuous) distributions with desirable properties for fitting purposes (i.e., presenting uni/multimodality, left/right/symmetrical skewness properties, different degrees of kurtosis, under/over dispersion features, wide panels of monotonic/non-monotonic hazard shapes, and so on). Most of them are defined by transforming a baseline cumulative distribution function (cdf) in a thorough way. Through the transformation technique, the pioneer remains the exp-G (E-G) family by [1] which consists in adding a new shape parameter; the corresponding cdf is defined by raising the baseline cdf to a (positive) power parameter. Deriving families include the notorious Kumaraswamy-G (K-G) family by [2], gamma-G (G-G) family by [3], Marshall-Olkin-G (MO-G) 
family by [4], the transmuted-G (T-G) family by [5], Beta-G (B-G) family by [6], the logistic-G (L-G) family by [7] and Weibull-G (W-G) family by [8]. For a more complete picture f the state-the-of-art, the interested reader is invited to consult the survey of [9].

Modern approaches to create new families of continuous distributions include the use of inverse (inverted) and/or truncated distributions as main generators. The logic of using inverse distributions is to exploit some of their specific properties to create original and flexible distributions, such as (i) their simplicity to use, (ii) their parsimony in parameter, (iii) their upside down bathtub (UBT) hazard rate properties, (iv) their ability to produce heavy tails (both (iii) and (iv) are generally not satisfied by the former (non-inverse) distribution when standard). New statistical models are thus derived, with practical benefit for the analysis of some data sets. For further detail on the inverse distributions, the reader is referred to [10-12]. Furthermore, several authors used the benefits of these inverse distributions for further perspectves, as the creation of general families of distributions, such as the inverse Weibull-G (IW-G) family by [13], type II general inverse exponential (TIIGIE) family by [14], generalized inverted Kumaraswamy-G (GIK-G) family by [15] and the generalized inverse Weibull-G (GIW-G) family by [16]. All these references demonstrate the success of the "inverse families" in many real-life applications dealing with data presenting various features (in terms of supports, skewness, kurtosis, uni/bimodal nature...). As an alternative or complementary approach, one can introduce new and simple families of continuous distributions by the truncation technique. The common scenario consists in truncating the cdf a flexible distribution (inverse or not, generally with support on $(0,+\infty)$ or $\mathbb{R})$ over the interval $(0,1)$ and compounding it by a simple baseline cdf. Current developments include the truncated Fréchet-G (TF-G) family by [17], truncated Burr-G (TB-G) family by [18], truncated Cauchy power-G (TCP-G) family by [19], truncated inverted Kumaraswamy-G (TIK-G) family by [20], and truncated inverse Weibull-G (TIW-G) family, also called type II truncated Fréchet-G (TIITF-G) family, by [21].

The novelty of the paper is centered around the TIW-G family by [21], justifying a retrospective on its construction. First of all, let us discuss the standard Weibull (W) distribution with scale parameter $a>0$ and shape parameter $b>0$. Basically, it is defined by the following cdf: $F_{1}(x ; a, b)=1-e^{-(a x)^{b}}, x>0$. It finds many statistical applications for the treatment of data exhibiting increasing, decreasing, and exponential failure rates, as commonly encountered in biology, medicine, physics, engineering, geology, quality control, economics, insurance, communication channels and social systems, among others. In this regard, we may refer to the unavoidable book by [22], as well as the survey by [23]. Then, several modifications and extensions have been proposed (see [23,24], and the references therein). The inverse Weibull (IW) distribution is one of these modifications, defined as the distribution of the reciprocal of a random variable following the standard $\mathrm{W}$ distribution. That is, the IW distribution with scale parameter $a>0$ and shape parameter $b>0$ is defined by the following cdf: $F_{2}(x ; a, b)=1-F_{1}(1 / x ; a, b)=e^{-(a / x)^{b}}, x>0$. Basically, it aims to produce a polynomial decay on the asymptotes on the corresponding pdf. In some sense, it makes the right tail of the former $\mathrm{W}$ distribution more heavier, allowing the analysis of some lifetime data having such a characteristic. Further interests and applications of the IW distributions are discussed in [25], and the references therein. On the other side, as a matter of fact, data can be the observations of variables with bounded range of possible values. This is particularly true in life-testing and reliability studies. Therefore, efforts have been made to introduce applicable truncated distributions, as the truncated W (TW) distribution by [26] and the truncated IW distribution (TIW) by [27]. In particular, the TIW distribution on $[0,1]$ with scale parameter 1 and shape parameter $b>0$ is defined by the following cdf: $F_{3}(x ; b)=F_{2}(x ; 1, b) / F_{2}(1 ; 1, b)=e^{1-x^{-b}}, x \in[0,1]$. Then, some authors used this simple and original one-parameter TIW distribution for another purpose: the construction of more general families of distributions. In particular, by using the type II composition technique derived to $[21,28]$ defined the TIW-G family by the following cdf:

$$
F_{*}(x ; b, \xi)=1-F_{3}(1-G(x ; \xi) ; b)=1-e^{1-(1-G(x ; \xi))^{-b}}, \quad x \in \mathbb{R},
$$


where $b>0$ is a shape parameter and $G(x ; \xi)$ denotes the cdf of a continuous baseline distribution with parameter vector $\xi$. The twin type I version of the TIW-G family can be found in [17] (with an appropriate configuration on the parameters). Furthermore, one can notice that, for $b=1$, the TIW-G family coincides with the W-G family under some configuration of the parameters.

In view of the work of [21], the advantages of the TIW-G family are the following ones: (i) the corresponding functions have tractable expressions, including the quantile function, (ii) there is only one additional parameter to the baseline distribution, (iii) several members of this family enjoy interesting properties, including various shapes for the corresponding functions, (iv) for some data sets, the TIW-G models can offer better alternatives to other well-established models, possibly defined with more parameters. In [21], these points are illustrated with the special member defined with the IW distribution as baseline. However, with a closer look on the shapes analysis, we see that a room of improvements exists: the hrf presents only monotonic curves; the bathtub and reverse bathtub shapes seem absent, as well as platykurtic and high heavy tail properties for the pdf.

With the idea in mind that the moderate lack of flexibility of the TIW-G family can be improved, we explore the solution provided by its exponentiated version, called the exponentiated TIW-G (ETIW-G) family. Thus, it is obtained from the E-G and TIW-G families; the corresponding cdf is defined by raising the cdf given by (1) to a positive power parameter. Such exponentiated extension remains an elegant way to enhance the flexible properties of an existing family. We thus follow the spirit of the constructions of the odd generalized exponential-G (OGE-G) family by [29], exponentiated T-G (ET-G) family by [30], type II power Topp-Leone-G (TIIPL-G) family by [31] and exponentiated power generalized Weibull power series-G (EPGWPS-G) family by [32], among others. On the other side, recent statistical works exploiting the benefit of adding a shape parameter to existing distributions can be found in $[24,33,34]$. Motivated by the exponentiated perspective, this paper is devoted to the complete study of the ETIW-G family, including its applicability in a statistical setting. We discuss the manner on how the new parameter can enhance the desirable properties of the former TIW-G family, mainly on the possible shapes of the corresponding probability functions. Furthermore, we show that the relationship between the complexity of the ETIW-G models and the gain in precision in terms of data fitting can be quite favorable. In particular, a focus is put on the special member defined with the IW distribution as baseline, corresponding to the exponentiated version of the special distribution considered in the applications of [21] and allowing fair comparisons in this regard. Among others, bathtub and reverse bathtub shapes for the hrf are observed, as well as platykurtic and high heavy tail properties for the pdf, opening new modelling facets.

The rest of this paper is organized as follows. In Section 2, we define the main probability functions of the ETIW-G family. Furthermore, we present a short list of special members of interest, including the one defined with the IW distribution as baseline. Some mathematical results on the new family are presented in Section 3, including stochastic ordering, uni/multimodality analysis, series expansions of crucial probability functions, probability weighted moments, raw and central moments, order statistics, and maximum likelihood method. Section 4 is devoted to the applied aspect of the ETIW-G family. In particular, we investigate the estimation of the model parameters by the maximum likelihood method by a numerical approach; a simulation is performed. Then, applications to three practical data sets are developed to illustrate the importance of the proposed models, including favorable comparisons with six well-reputed models. The paper is concluded in Section 5.

\section{The ETIW-G Family}

The presentation and prime interests of the ETIW-G family are the objects of this section. 


\subsection{Probability Functions}

As formally described in the introduction, the ETIW-G family is defined by the following cdf:

$$
F(x ; a, b, \xi)=\left(1-e^{1-(1-G(x ; \xi))^{-b}}\right)^{a}, \quad x \in \mathbb{R},
$$

where $a>0$ and $b>0$ are both shape parameters and $G(x ; \xi)$ is the cdf of a continuous baseline distribution, with $\xi$ as parameter vector. When $a=1$, we rediscover the TIW-G family (or TIITF-G family) introduced by [21]. Furthermore, when $b=1$, the ETIW-G family coincides with the OGE-G family under some configuration of the parameters.

The pdf of the the ETIW-G family is given by

$$
f(x ; a, b, \xi)=\operatorname{abg}(x ; \xi)(1-G(x ; \xi))^{-b-1} e^{1-(1-G(x ; \xi))^{-b}}\left(1-e^{1-(1-G(x ; \xi))^{-b}}\right)^{a-1}, \quad x \in \mathbb{R},
$$

where $g(x ; \xi)$ is the corresponding pdf of $G(x ; \xi)$.

Furthermore, the survival function, say $R(x ; a, b, \xi)$, hazard rate function (hrf), say $h(x ; a, b, \xi)$, reversed hazard rate function, say $\tau(x ; a, b, \xi)$, and cumulative hazard rate function, say $H(x ; a, b, \xi)$ are, respectively, given by

$$
\begin{gathered}
R(x ; a, b, \xi)=1-\left(1-e^{1-(1-G(x ; \xi))^{-b}}\right)^{a}, \quad x \in \mathbb{R}, \\
h(x ; a, b, \xi)=a b g(x ; \xi)(1-G(x ; \xi))^{-b-1} e^{1-(1-G(x ; \xi))^{-b}} \frac{\left(1-e^{1-(1-G(x ; \xi))^{-b}}\right)^{a-1}}{1-\left(1-e^{1-(1-G(x ; \xi))^{-b}}\right)^{a}}, \quad x \in \mathbb{R}, \\
\tau(x ; a, b, \xi)=\frac{a b g(x ; \xi)(1-G(x ; \xi))^{-b-1} e^{1-(1-G(x ; \xi))^{-b}}}{1-e^{1-(1-G(x ; \xi))^{-b}}, \quad x \in \mathbb{R}}
\end{gathered}
$$

and

$$
H(x ; a, b, \xi)=-\log \left[1-\left(1-e^{1-(1-G(x ; \xi))^{-b}}\right)^{a}\right], \quad x \in \mathbb{R} .
$$

When the baseline distribution is a lifetime distribution, these functions play important roles in survival analysis, with a multitude of applications. In this regard, we may refer the reader to [35].

Finally, the quantile function (qf) of the ETIW-G family is obtained by inverting (2). After some developments, we arrive at the following function:

$$
Q(u ; a, b, \xi)=Q_{G}\left(1-\left[1-\log \left(1-u^{1 / a}\right)\right]^{-1 / b} ; \xi\right), \quad u \in(0,1),
$$

where $Q_{G}(u ; \xi)$ is the corresponding qf of $G(x ; \xi)$. Thus, thanks to the closed-form expression of $Q(u ; a, b, \xi)$, the quartiles can be expressed, and the inverse transform sampling can be applied to generate numbers of the related distribution. Furthermore, the qf appears in the definitions of some measures of skewness and kurtosis having the merits to exist in all circumstance, i.e., whatever the baseline distribution. We may refer to the Bowley measure of skewness and the Moors measure of kurtosis (see [36,37], respectively)

\subsection{Some Special Members of the ETIW-G Family}

The ETIW-G family contains a plethora of distributions (with various supports, characteristics...). A short list of them, defined with standard baseline distributions, are presented in Table 1. 
In Table 1, the following notations have been introduced. For the ETIWG distribution, we have set $\gamma(\alpha, \beta x)=\int_{0}^{\beta x} t^{\alpha-1} e^{-t} d t, \Gamma(\alpha)=\int_{0}^{+\infty} t^{\alpha-1} e^{-t} d t$, and for the ETIWN distribution, we have set $\Phi(x ; \mu, \sigma)=\int_{-\infty}^{x}\left(1 / \sqrt{2 \pi \sigma^{2}}\right) e^{-(t-\mu)^{2} /\left(2 \sigma^{2}\right)} d t$.

Table 1. A short list of special members of the ETIW-G family.

\begin{tabular}{cccccc}
\hline ETIW-G & Baseline Distribution & $\xi$ & Support & $G(x ; \xi)$ & $\boldsymbol{F}(\boldsymbol{x} ; \boldsymbol{a}, \boldsymbol{b}, \boldsymbol{\xi})$ \\
\hline ETIWU & Uniform & $(\theta)$ & $(0, \theta)$ & $x / \theta$ & $\left(1-e^{\left.1-(1-x / \theta)^{-b}\right)^{a}}\right.$ \\
\hline ETIWP & Power & $(a)$ & $(0,1)$ & $x^{\alpha}$ & $\left(1-e^{\left.1-\left(1-x^{\alpha}\right)^{-b}\right)^{a}}\right.$ \\
\hline \multirow{2}{*}{ ETIWD } & Dagum & $(\alpha, \beta, \gamma)$ & $(0,+\infty)$ & $\left(1+(x / \alpha)^{-\beta}\right)^{-\gamma}$ & $\left(1-e^{\left.1-\left[1-\left(1+(x / \alpha)^{-\beta}\right)^{-\gamma}\right]^{-b}\right)^{a}}\right.$ \\
\hline ETIWLi & Lindley & $(\theta)$ & $(0,+\infty)$ & $1-(1+\theta+\theta x) e^{-\theta x} /(1+\theta)$ & $\left(1-e^{\left.1-(1+\theta)^{b}(1+\theta+\theta x)^{-b} e^{b \theta x}\right)^{a}}\right.$ \\
\hline ETIWHC & Half Cauchy & $(\alpha)$ & $(0,+\infty)$ & $(2 / \pi) \arctan (x / \alpha)$ & $\left(1-e^{\left.1-(1-(2 / \pi) \arctan (x / \alpha))^{-b}\right)^{a}}\right.$ \\
\hline ETIWG & Gamma & $(\alpha, \beta)$ & $(0,+\infty)$ & $\gamma(\alpha, \beta x) / \Gamma(\alpha)$ & $\left(1-e^{\left.1-(1-\gamma(\alpha, \beta x) / \Gamma(\alpha))^{-b}\right)^{a}}\right.$ \\
\hline ETIWLo & Logistic & $(\alpha, \beta)$ & $\mathbb{R}$ & $\left(1+e^{-(x-\alpha) / \beta}\right)$ & $\left(1-e^{1-\left[1-\left(1+e^{-(x-\alpha) / \beta}\right)^{-1}\right]^{-b}}\right)^{a}$ \\
\hline ETIWN & Normal & $(\mu, \sigma)$ & $\mathbb{R}$ & $\Phi(x ; \mu, \sigma)$ & $\left(1-e^{\left.1-(1-\Phi(x ; \mu, \sigma))^{-b}\right)^{a}}\right.$ \\
\hline
\end{tabular}

\subsection{The ETIWIW Distribution}

After preliminary analyzes, thanks to an interesting compromise between simplicity and statistical flexibility in a wide sense, we put the light on the special member of the ETIW-G family defined with the IW distribution as baseline. Furthermore, it corresponds to the exponentiated version of the special distribution considered by Section 4.3 of [21]. We logically call it the ETIWIW distribution.

First of all, we recall that the IW distribution is defined by the following cdf:

$$
G(x ; \mu, \theta)=e^{-(\mu / x)^{\theta}}, \quad g(x ; \mu, \theta)=\theta \mu^{\theta} x^{-\theta-1} e^{-(\mu / x)^{\theta}}, \quad x>0,
$$

where $\mu>0$ is a scale parameter and $\theta>0$ is a shape parameter (hence, $\xi=(\mu, \theta)$ ). Motivations and applications of the IW distribution can be found in [25], and the references therein.

By inserting the expression of $G(x ; \mu, \theta)$ into (2), the ETIWIW distribution is defined by the cdf given by

$$
F(x ; a, b, \mu, \theta)=\left(1-e^{1-\left[1-e^{-(\mu / x)^{\theta}}\right]^{-b}}\right)^{a}, \quad x>0
$$

Furthermore, based on (3) and (4), the corresponding pdf and hrf are, respectively, given by

$$
\begin{aligned}
& f(x ; a, b, \mu, \theta)= \\
& a b \theta \mu^{\theta} x^{-\theta-1} e^{-(\mu / x)^{\theta}}\left[1-e^{-(\mu / x)^{\theta}}\right]^{-b-1} e^{1-\left[1-e^{-(\mu / x)^{\theta}}\right]^{-b}}\left(1-e^{1-\left[1-e^{-(\mu / x)^{\theta}}\right]^{-b}}\right)^{a-1}, \quad x>0
\end{aligned}
$$

and

$$
\begin{aligned}
& h(x ; a, b, \mu, \theta)= \\
& a b \theta \mu^{\theta} x^{-\theta-1} e^{-(\mu / x)^{\theta}}\left[1-e^{-(\mu / x)^{\theta}}\right]^{-b-1} e^{1-\left[1-e^{-(\mu / x)^{\theta}}\right]^{-b}} \frac{\left(1-e^{1-\left[1-e^{-(\mu / x)^{\theta}}\right]^{-b}}\right)^{a-1}}{1-\left(1-e^{1-\left[1-e^{-(\mu / x)^{\theta}}\right]^{-b}}\right)^{a},} x>0 .
\end{aligned}
$$

The flexibility of the shapes of $f(x ; a, b, \mu, \theta)$ and $h(x ; a, b, \mu, \theta)$ is illustrated in Figures 1 and 2. 
In Figure 1 , we see that $f(x ; a, b, \mu, \theta)$ enjoys reverse J, left skewed, symmetrical and right skewed shapes, various platykurtic, letokurtic and mesokurtic shapes, and various degree of heaviness on the tails as well. In particular, the parameter $a$ has a strong effect on the skewness properties of the distribution, whereas $\mu$ seems to have a greatest impact on the kurtosis. In Figure 2, we see that $h(x ; a, b, \mu, \theta)$ possesses increasing, decreasing, bathtub and upside down bathtub shapes. The parameters $a$ and $\theta$ seem to have a great influence in this regards. Thanks to the non-monotonic shapes of the hrf, as well as platykurtic and high heavy tail properties for the pdf, the overall flexibility of the former TIWIW distribution is improved (see Figure 3 of [21]). Furthermore, the wide variety of shapes of these functions gives a strong indicator on the usefulness of the ETIWIW model for lifetime data fitting purposes, hoping enough ability to capture local phenomena. This point will be investigated with success in Sections 4.2-4.4.

Furthermore, the qf of the ETIWIW distribution has a closed-form, and can be expressed as

$$
Q(u ; a, b, \mu, \theta)=\mu\left\{-\log \left(1-\left[1-\log \left(1-u^{1 / a}\right)\right]^{-1 / b}\right)\right\}^{-1 / \theta}, \quad u \in(0,1) .
$$

The median of the ETIWIW distribution can be derived as $M_{0.5}=Q(0.5 ; a, b, \mu, \theta)$, as well as the two other quartiles, i.e., $M_{0.25}=Q(0.25 ; a, b, \mu, \theta)$ and $M_{0.75}=Q(0.75 ; a, b, \mu, \theta)$. Furthermore, we can apply the inverse transform sampling to generate numbers of the ETIWIW distribution. This method will be performed in Section 4.1 in the context of a simulation study.
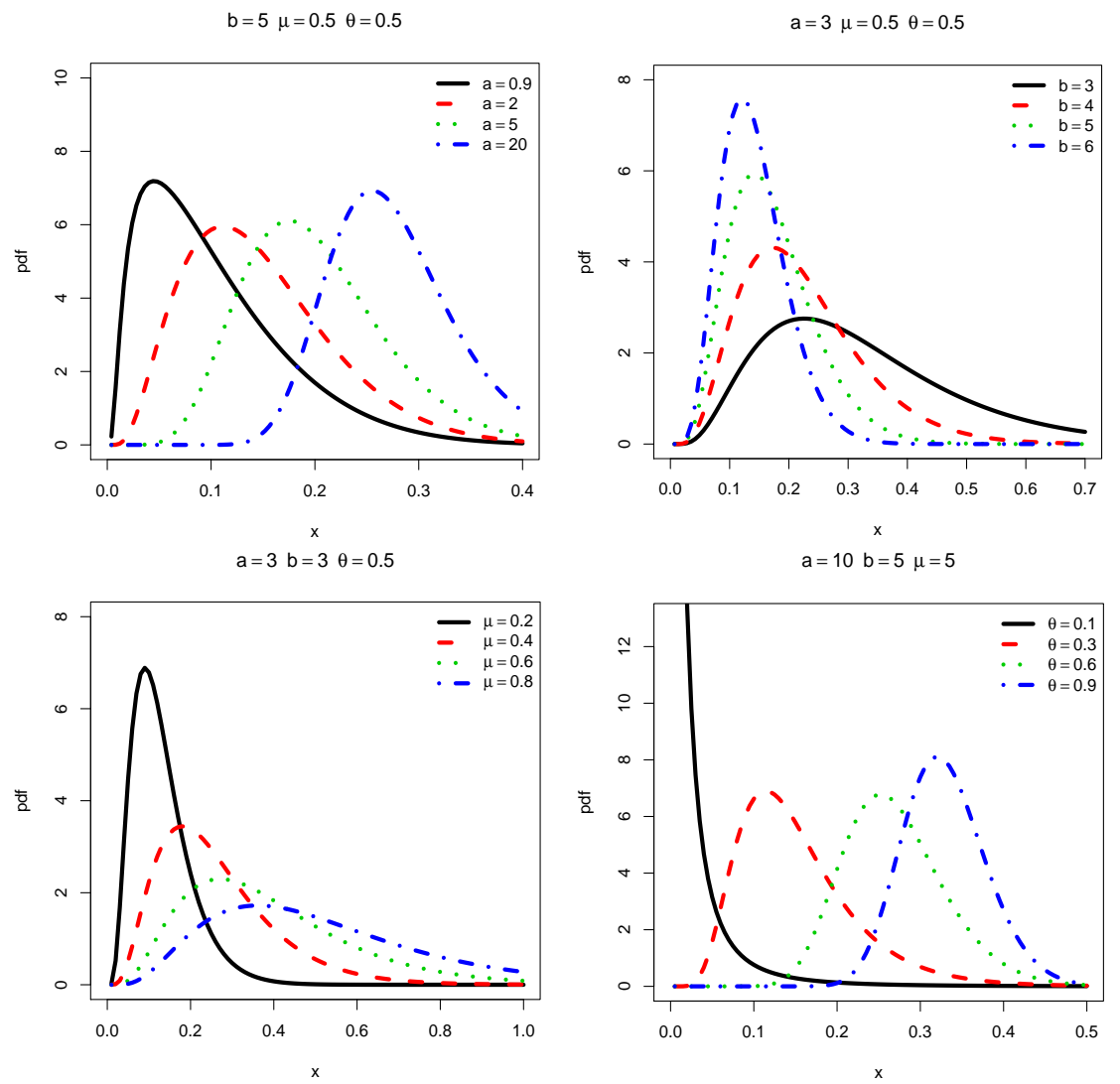

Figure 1. Plots for the pdf of the ETIWIW distribution for various values of the parameters. 

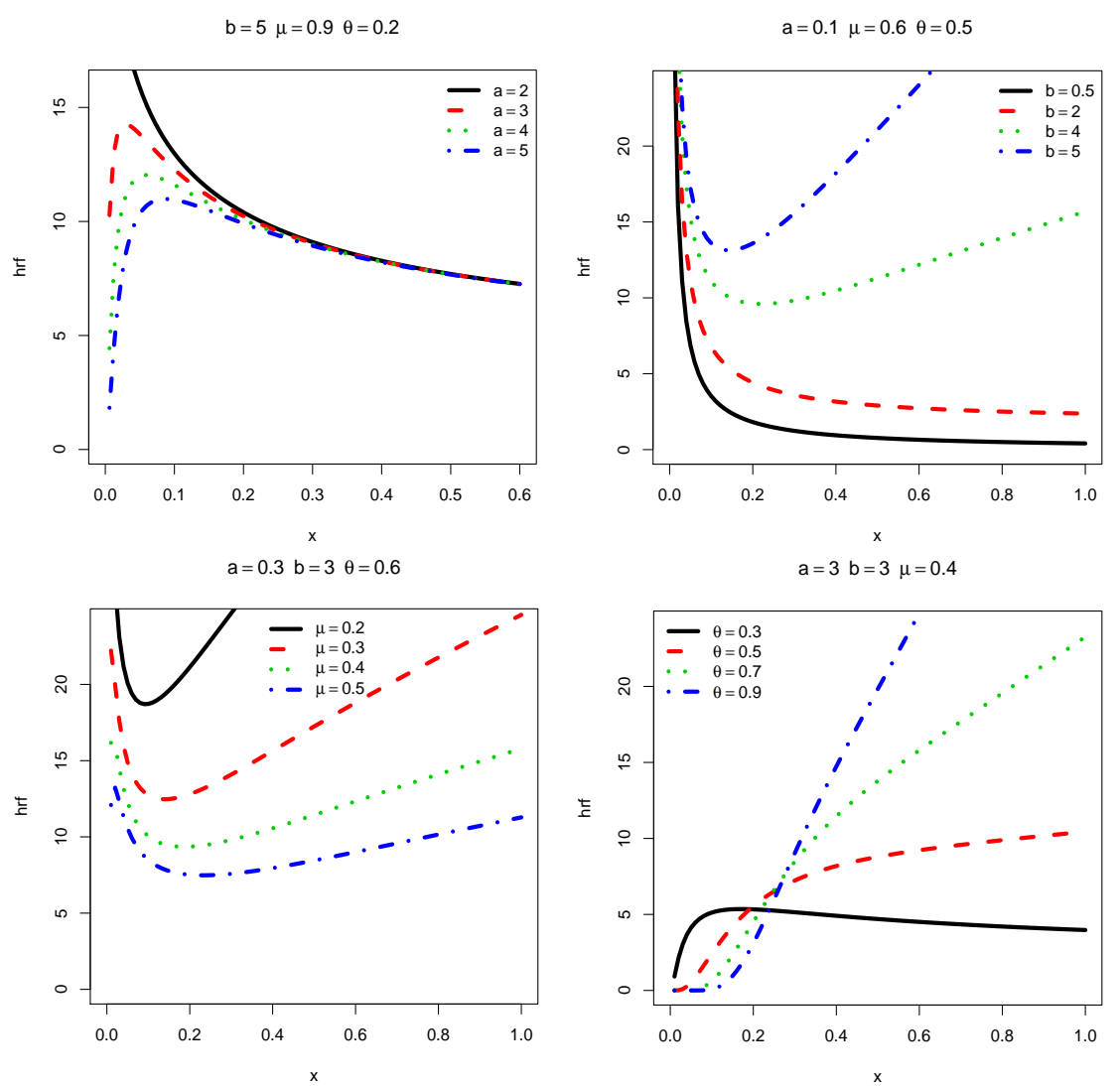

Figure 2. Plots for the hrf of the ETIWIW distribution for various values of the parameters.

\section{Mathematical Investigations}

Hereafter, we consider a random variable $X$ having the cdf of the ETIW-G family given by (2). Furthermore, it is supposed that all the introduced quantities exist, which is not guarantee a priori, depending on the definition of the baseline distribution mainly.

\subsection{Stochastic Ordering Results}

The following proposition presents simple stochastic ordering results involving the cdf of the ETIW-G family.

Proposition 1. The following stochastic ordering results hold.

- Let $G_{1}(x ; \xi)$ and $G_{2}(x ; \xi)$ be two baseline cdfs, and $F_{G}(x ; a, b, \xi)$ be defined as (2). Then, if $G_{2}(x ; \xi) \geq$ $G_{1}(x ; \xi)$, for any $x \in \mathbb{R}$, we have

$$
F_{G_{1}}(x ; a, b, \xi) \leq F_{G_{2}}(x ; a, b, \xi)
$$

- For any $b_{*} \geq b>0$ and $a_{*} \geq a>0$, and any $x \in \mathbb{R}$, we have

$$
F\left(x ; a_{*}, b, \xi\right) \leq F\left(x ; a, b_{*}, \xi\right)
$$

Proof. Let us prove the two points, in turn.

- The desired inequality follows from the fact that $F_{G}(x ; a, b, \xi)=F_{\diamond}(G(x ; \xi) ; a, b)$, where $F_{\diamond}(x ; a, b)=\left(1-e^{1-(1-x)^{-b}}\right)^{a}, x \in(0,1)$, is an increasing function with respect to $x$ (as the cdf of the exponentiated type II truncated inverse Weibull distribution over $(0,1)$ ). 
- Since $G(x ; \xi) \in(0,1)$ (excluding the limit cases), $b_{*} \geq b>0$ and $a_{*} \geq a>0$, we have the following chain of equivalences:

$$
\begin{aligned}
& (1-G(x ; \xi))^{b_{*}} \leq(1-G(x ; \xi))^{b} \Leftrightarrow(1-G(x ; \xi))^{-b} \leq(1-G(x ; \xi))^{-b_{*}} \\
& \Leftrightarrow \quad 1-(1-G(x ; \xi))^{-b_{*}} \leq 1-(1-G(x ; \xi))^{-b} \Leftrightarrow e^{1-(1-G(x ; \xi))^{-b *}} \leq e^{1-(1-G(x ; \xi))^{-b}} \\
& \Leftrightarrow \quad 1-e^{1-(1-G(x ; \xi))^{-b}} \leq 1-e^{1-(1-G(x ; \xi))^{-b *}} \Leftrightarrow \\
& \Leftrightarrow \quad\left(1-e^{1-(1-G(x ; \xi))^{-b}}\right)^{a_{*}} \leq\left(1-e^{1-(1-G(x ; \xi))^{-b}}\right)^{a} \leq\left(1-e^{1-(1-G(x ; \xi))^{-b *}}\right)^{a}
\end{aligned}
$$

implying that $F\left(x ; a_{*}, b, \xi\right) \leq F\left(x ; a, b_{*}, \xi\right)$.

This ends the proof of Proposition 1.

In particular, by taking $a=1$ and $b_{*}=b$, the first point of Proposition 1 allows us to compare the cdf of the ETIW-G family and the one of the TIW-G family by [21], i.e., given by (1). Indeed, if $a \leq 1$, we have $F_{*}(x ; b, \xi) \leq F(x ; a, b, \xi)$ and if $a>1$, we have $F_{*}(x ; b, \xi)>F(x ; a, b, \xi)$. These simple inequalities show that the ETIW-G family can reach different target in terms of modelling in comparison to the former TIW-G family.

\subsection{Uni/multimodality Analysis}

A basic uni/multimodality analysis of a member of the ETIW-G family can be investigated analytically. In this regard, let us set $Y(x)=\log [f(x ; a, b, \xi)]$. Then, a mode for $f(x ; a, b, \xi)$ is a solution of the following non-linear equation: $f(x ; a, b, \xi)^{\prime}=0$ or, equivalently, $\mathrm{Y}(x)^{\prime}=0$, where the derivative is taken with respect to $x$. After some algebraic manipulations, one can express $\mathrm{Y}(x)^{\prime}$ as

$$
Y(x)^{\prime}=\frac{g(x ; \xi)^{\prime}}{g(x ; \xi)}+(b+1) \frac{g(x ; \xi)}{1-G(x ; \xi)}-b g(x ; \xi)(1-G(x ; \xi))^{-b-1}\left[1-\frac{a-1}{e^{(1-G(x ; \xi))^{-b}-1}-1}\right] .
$$

If there is multiple modes, the considered distribution has a multimodal feature. The nature of a mode, say $x_{m}$, can be investigated by studying the sign of $Y(x)^{\prime \prime}$, where

$$
\begin{aligned}
Y(x)^{\prime \prime} & =\frac{g(x ; \xi)^{\prime \prime} g(x ; \xi)-\left[g(x ; \xi)^{\prime}\right]^{2}}{[g(x ; \xi)]^{2}}+(b+1) \frac{g(x ; \xi)^{\prime}(1-G(x ; \xi))+g(x ; \xi)^{2}}{[1-G(x ; \xi)]^{2}} \\
& -b\left[g(x ; \xi)^{\prime}(1-G(x ; \xi))^{-b-1}+(b+1)[g(x ; \xi)]^{2}(1-G(x ; \xi))^{-b-2}\right]\left[1-\frac{a-1}{e^{(1-G(x ; \xi))^{-b}-1}-1}\right] \\
& -b^{2}(a-1) g(x ; \xi)(1-G(x ; \xi))^{-b-1} \frac{e^{(1-G(x ; \xi))^{-b}-1} g(x ; \xi)(1-G(x ; \xi))^{-b-1}}{\left(e^{(1-G(x ; \xi))^{-b}-1}-1\right)^{2}} .
\end{aligned}
$$

Then, $x_{m}$ is a local maximum if $Y\left(x_{m}\right)^{\prime \prime}<0$, it is a local minimum if $Y\left(x_{m}\right)^{\prime \prime}>0$ and it is a point of inflexion if $Y\left(x_{m}\right)^{\prime \prime}=0$.

In the context of the ETIWIW distribution (i.e., defined with the cdf given by (5)), we have $\xi=(\mu, \theta), G(x ; \mu, \theta)=e^{-(\mu / x)^{\theta}}$ with $x>0, g(x ; \mu, \theta)=\theta \mu^{\theta} x^{-\theta-1} e^{-(\mu / x)^{\theta}}$, and

$$
g(x ; \mu, \theta)^{\prime}=\theta \mu^{\theta} x^{-\theta-2} e^{-(\mu / x)^{\theta}}\left[\theta\left(\frac{\mu}{x}\right)^{\theta}-\theta-1\right]
$$

and

$$
g(x ; \mu, \theta)^{\prime \prime}=\theta \mu^{\theta} x^{-\theta-3} e^{-(\mu / x)^{\theta}}\left[\theta^{2}\left(\frac{\mu}{x}\right)^{2 \theta}-3 \theta^{2}\left(\frac{\mu}{x}\right)^{\theta}-3 \theta\left(\frac{\mu}{x}\right)^{\theta}+\theta^{2}+3 \theta+2\right] .
$$


In view of the complexity of the above equations, closed-form(s) for the mode(s) (non reduced to 0 ) is(are) difficult to obtain. However, for given parameters $a, b$ and $G(x ; \xi)$, one can determine it(them) by the use of any mathematical software (R, Python, Mapple, Mathematica...).

\subsection{Tractable Series Expansions}

The following result investigates a tractable series expansion for the following function: $f(x ; a, b, \xi) F(x ; a, b, \xi)^{h}$ with $h \geq 0$. It is expressed in terms of pdfs of the exp-G family by [1]. From the computational point of view, the use of such expansions can be more efficient than compute directly integrals involving $f(x ; a, b, \xi) F(x ; a, b, \xi)^{h}$, which are legion in probability theory.

Proposition 2. For any $\gamma>0$, let $\pi_{\gamma}(x ; \xi)=\gamma g(x ; \xi) G(x ; \xi)^{\gamma-1}$, which corresponds to the pdf of the exp-G family with power parameter $\gamma>0$. Then, for any $h \geq 0$, we have the following series expansion:

$$
f(x ; a, b, \xi) F(x ; a, b, \xi)^{h}=\sum_{k, \ell=0}^{+\infty} \sum_{m=1}^{+\infty} v_{k, \ell, m}^{[h]} \pi_{m}(x ; \xi)
$$

where

$$
v_{k, \ell, m}^{[h]}=\frac{1}{h+1}\left(\begin{array}{c}
a(h+1) \\
k
\end{array}\right)\left(\begin{array}{c}
-b \ell \\
m
\end{array}\right)(-1)^{k+\ell+m} e^{k} \frac{1}{\ell !} k^{\ell}
$$

and, for any $u \in \mathbb{N}$ and $v \in \mathbb{R},\left(\begin{array}{l}v \\ u\end{array}\right)=v(v-1) \ldots(v-u+1) / u$ !.

Proof. We first investigate a series expansion for $F(x ; a, b, \xi)^{h+1}$, and will obtain the desired result upon differentiation with respect to $x$. Let $\Pi_{\gamma}(x ; \xi)=G(x ; \xi)^{\gamma}$ be the cdf of the exp-G family with power parameter $\gamma>0$ (the one with the corresponding pdf given by $\pi_{\gamma}(x ; \xi)$ ). For the special case $\gamma=0$, we set $\Pi_{0}(x ; \xi)=1$. It follows from the generalized binomial formula that

$$
F(x ; a, b, \xi)^{h+1}=\left(1-e^{1-(1-G(x ; \xi))^{-b}}\right)^{a(h+1)}=\sum_{k=0}^{+\infty}\left(\begin{array}{c}
a(h+1) \\
k
\end{array}\right)(-1)^{k} e^{k} e^{-k(1-G(x ; \xi))^{-b}} .
$$

Now, by virtue of the series expansion of the exponential function, we get

$$
e^{-k(1-G(x ; \xi))^{-b}}=\sum_{\ell=0}^{+\infty} \frac{1}{\ell !}(-1)^{\ell} k^{\ell}(1-G(x ; \xi))^{-b \ell} .
$$

By applying again the generalized binomial formula, we have

$$
(1-G(x ; \xi))^{-b \ell}=\sum_{m=0}^{+\infty}\left(\begin{array}{c}
-b \ell \\
m
\end{array}\right)(-1)^{m} \Pi_{m}(x ; \xi)
$$

By putting the above equalities together, we obtain

$$
F(x ; a, b, \xi)^{h+1}=\sum_{k, \ell, m=0}^{+\infty}\left(\begin{array}{c}
a(h+1) \\
k
\end{array}\right)\left(\begin{array}{c}
-b \ell \\
m
\end{array}\right)(-1)^{k+\ell+m} e^{k} \frac{1}{\ell !} k^{\ell} \Pi_{m}(x ; \xi) .
$$

Then, upon differentiation with respect to $x$, we get

$$
f(x ; a, b, \xi) F(x ; a, b, \xi)^{h}=\frac{1}{h+1}\left\{F(x ; a, b, \xi)^{h+1}\right\}^{\prime}=\sum_{k, \ell=0}^{+\infty} \sum_{m=1}^{+\infty} v_{k, \ell, m}^{[h]} \pi_{m}(x ; \xi) .
$$

This ends the proof of Proposition 2. 
Furthermore, one can remark that, for $h=0$, Proposition 2 gives a series expansion for $f(x ; a, b, \xi)$. Also, we have

$$
f(x ; a(h+1), b, \xi)=\sum_{k, \ell=0}^{+\infty} \sum_{m=1}^{+\infty} w_{k, \ell, m}^{[h]} \pi_{m}(x ; \xi),
$$

where $w_{k, \ell, m}^{[h]}=(h+1) v_{k, \ell, m}^{[h]}$.

In the framework of the ETIWIW distribution, Proposition 2 means that $f(x ; a, b, \mu, \theta) F(x ; a, b, \mu, \theta)^{h}$ (and $f(x ; a, b, \mu, \theta)$ in particular) can be expressed as an infinite linear combination of pdfs of the exponentiated IW distribution introduced by [25].

In the next, Proposition 2 is useful to provide analytical series expansion for some important measures, as moments.

\subsection{Probability Weighted Moments}

The probability weighted moments have the following interests: they generalize the notions of raw moments, which naturally appear in the theory of order statistics and have numerous issues for parameter estimation purposes (as in the so-called method of probability weighted moments introduced by [38]). Modern developments can be found in [39], and the references therein.

Here, the $(r, h)^{\text {th }}$ probability weighted moment of $X$ is defined by

$$
\tau_{r, h}=E\left(X^{r} F(X ; a, b, \xi)^{h}\right)=\int_{-\infty}^{\infty} x^{r} F(x ; a, b, \xi)^{h} f(x ; a, b, \xi) d x .
$$

In full generality, standard methods fall to determine it in a simple manner. However, it can be computed numerically with reasonable errors by the use of any mathematical software. Alternatively, as an analytical approach, it follows from Proposition 2 that

$$
\tau_{r, h}=\sum_{k, \ell=0}^{+\infty} \sum_{m=1}^{+\infty} v_{k, \ell, m}^{[h]} \mu_{r, m}^{*}
$$

where $\mu_{r, m}^{*}=\int_{-\infty}^{\infty} x^{r} \pi_{m}(x ; \xi) d x=m \int_{0}^{1} u^{m-1} Q_{G}(u ; \xi)^{r} d u$, which corresponds to the $r^{\text {th }}$ raw moment of a random variable having the cdf of the exp-G family with power parameter $m$.

If $G(x ; \xi)$ is of moderate complexity (as any scholar cdf), one can express $\mu_{r, m}^{*}$ in an easy way, and consider the following tractable series approximation for $\tau_{r, h}$ :

$$
\tau_{r, h} \approx \sum_{k, \ell=0}^{M} \sum_{m=1}^{M} v_{k, \ell, m}^{[h]} \mu_{r, m}^{*}
$$

where $M$ denotes a large integer, say $M=50$.

For instance, in the context of the ETIWIW distribution, after some algebraic manipulations, we arrive at $\mu_{r, m}^{*}=m^{r / \theta} \mu^{r} \Gamma(1-r / \theta)$ (see also Equation (5) of [25]).

\subsection{Raw and Central Moments, with Applications}

The raw moments from $X$ can be viewed as special cases of the probability weighted moments; the $r^{\text {th }}$ raw moment of $X$ corresponds to the case $h=0$, i.e., $\mu_{r}^{\prime}=E\left(X^{r}\right)=\tau_{r, 0}$. Then, we easily derive the mean and variance of $X$; they are, respectively, given by $\mu=\mu_{1}^{\prime}$ and $\sigma^{2}=\mu_{2}^{\prime}-\mu^{2}$. With a direct application of the standard binomial formula, we also get the $r^{\text {th }}$ central moment of $X$ as

$$
\mu_{r}=E\left[(X-\mu)^{r}\right]=\sum_{k=0}^{r}\left(\begin{array}{l}
r \\
k
\end{array}\right)(-1)^{k} \mu^{k} \mu_{r-k}^{\prime} .
$$


We can deduce the coefficient of variation given by $C V=\sigma / \mu$. Furthermore, the $r^{\text {th }}$ general coefficient of $X$ can be expressed as $C_{r}=\mu_{r} / \sigma^{r / 2}$. As standard measures of skewness and kurtosis, one can consider the coefficients $C_{3}$ and $C_{4}$, respectively. The sign of $C_{3}$ informs us on the skewed nature of the distribution (left if $C_{3}<0$, symmetrical if $C_{3}=0$ or right if $C_{3}>0$ ). Furthermore, the value of $C_{4}$ measures the "tailedness" of the distribution.

\subsection{Order Statistics}

Order statistics are used in numerous applications in statistical estimation and reliability analysis. The related theory is well-known; it can be found in [40], for instance. Here, we present the basics of the order statistics in the context of the ETIW-G family. Let $X_{1}, \ldots, X_{n}$ be a random sample of size $n$ from $X$ and $X_{i: n}$ be the $i^{\text {th }}$ order statistic, i.e., $X_{1: n}=\inf \left(X_{1}, \ldots, X_{n}\right), X_{2: n}=\inf \left(\left\{X_{1}, \ldots, X_{n}\right\} /\left\{X_{1: n}\right\}\right)$, $X_{3: n}=\inf \left(\left\{X_{1}, \ldots, X_{n}\right\} /\left\{X_{1: n}, X_{2: n}\right\}\right), \ldots$ and $X_{n: n}=\sup \left(X_{1}, \ldots, X_{n}\right)$.

The following result is about a distributional property of $X_{i: n}$.

Proposition 3. The cdf of $X_{i: n}$ can be expressed as as linear combination of cdfs of the ETIW-G family. Indeed, we have

$$
F_{i: n}(x ; a, b, \xi)=\sum_{k=i}^{n} \sum_{j=0}^{n-k}\left(\begin{array}{l}
n \\
k
\end{array}\right)\left(\begin{array}{c}
n-k \\
j
\end{array}\right)(-1)^{j} F(x ; a(k+j), b, \xi), \quad x \in \mathbb{R} .
$$

Proof. By using a well-known formula on the cdf of the $i^{\text {th }}$ order statistic (see [40]), applying the standard binomial formula and noticing that $F(x ; a, b, \xi)^{h}=\left(1-e^{1-(1-G(x ; \xi))^{-b}}\right)^{a h}=F(x ; a h, b, \xi)$, we get

$$
\begin{aligned}
F_{i: n}(x ; a, b, \xi) & =\sum_{k=i}^{n}\left(\begin{array}{l}
n \\
k
\end{array}\right) F(x ; a, b, \xi)^{k}(1-F(x ; a, b, \xi))^{n-k} \\
& =\sum_{k=i}^{n} \sum_{j=0}^{n-k}\left(\begin{array}{l}
n \\
k
\end{array}\right)\left(\begin{array}{c}
n-k \\
j
\end{array}\right)(-1)^{j} F(x ; a, b, \xi)^{k+j} \\
& =\sum_{k=i}^{n} \sum_{j=0}^{n-k}\left(\begin{array}{l}
n \\
k
\end{array}\right)\left(\begin{array}{c}
n-k \\
j
\end{array}\right)(-1)^{j} F(x ; a(k+j), b, \xi) .
\end{aligned}
$$

This proves Proposition 3.

As an immediate consequence of Proposition 3, upon differentiation of $F_{i: n}(x ; a, b, \xi)$ with respect to $x$, the pdf of $X_{i: n}$ can be expressed as as linear combination of pdfs of the ETIW-G family:

$$
f_{i: n}(x ; a, b, \xi)=\sum_{k=i}^{n} \sum_{j=0}^{n-k}\left(\begin{array}{l}
n \\
k
\end{array}\right)\left(\begin{array}{c}
n-k \\
j
\end{array}\right)(-1)^{j} f(x ; a(k+j), b, \xi), \quad x \in \mathbb{R} .
$$

In particular, by using the equality above and (7), the $r^{\text {th }}$ raw moment of $X_{i: n}$ can be expressed as

$$
\mu_{r, i: n}^{\prime}=E\left(X_{i: n}^{r}\right)=\sum_{k=i}^{n} \sum_{j=0}^{n-k} \sum_{k_{*}, \ell=0}^{+\infty} \sum_{m=1}^{+\infty} \omega_{k_{*}, \ell, m}^{[k+j-1]} \mu_{r, m}^{*},
$$

where $\omega_{k_{*}, \ell, m}^{[k+j-1]}=\left(\begin{array}{c}n \\ k\end{array}\right)\left(\begin{array}{c}n-k \\ j\end{array}\right)(-1)^{j} w_{k_{*}, \ell, m}^{[k+j-1]}$, with an acceptable approximation by substituting infinity by any large integer. We thus can derive the standard central, dispersion, skewness and kurtosis parameters for $X_{i: n}$, and so on. 


\subsection{Maximum Likelihood Method}

Here, the estimation of the parameters of the ETIW-G model is investigated by the maximum likelihood method for complete samples. Let $x_{1}, \ldots, x_{n}$ be observed values from a random variable having the cdf given by (2), with parameters $b, a$ and $\xi$. Then, the log-likelihood function for $(a, b, \xi)$ is obtained as

$$
\begin{aligned}
\ell(a, b, \xi) & =n \log (a)+n \log (b)+\sum_{i=1}^{n} \log \left[g\left(x_{i} ; \xi\right)\right]-(b+1) \sum_{i=1}^{n} \log \left[1-G\left(x_{i} ; \xi\right)\right]+n \\
& -\sum_{i=1}^{n}\left(1-G\left(x_{i} ; \xi\right)\right)^{-b}+(a-1) \sum_{i=1}^{n} \log \left[1-e^{1-\left(1-G\left(x_{i} ; \xi\right)\right)^{-b}}\right]
\end{aligned}
$$

Then, upon differentiation of $\ell(a, b, \xi)$ according to each of the parameter-variable, the components of the score function $U(a, b, \xi)=\left(U_{b}, U_{a}, U_{\xi}\right)$ are given by

$$
\begin{gathered}
U_{a}=\frac{n}{a}+\sum_{i=1}^{n} \log \left[1-e^{1-\left(1-G\left(x_{i} ; \xi\right)\right)^{-b}}\right] \\
U_{b}=\frac{n}{b}-\sum_{i=1}^{n} \log \left[1-G\left(x_{i} ; \xi\right)\right]+\sum_{i=1}^{n}\left(1-G\left(x_{i} ; \xi\right)\right)^{-b} \log \left(1-G\left(x_{i} ; \xi\right)\right) \\
-(a-1) \sum_{i=1}^{n} \frac{\left(1-G\left(x_{i} ; \xi\right)\right)^{-b} \log \left(1-G\left(x_{i} ; \xi\right)\right)}{e^{\left(1-G\left(x_{i} ; \xi\right)\right)^{-b}-1}-1}
\end{gathered}
$$

and

$$
\begin{aligned}
U_{\xi} & =\sum_{i=1}^{n} \frac{g\left(x_{i} ; \xi\right) \xi}{g\left(x_{i} ; \xi\right)}+(b+1) \sum_{i=1}^{n} \frac{G\left(x_{i} ; \xi\right) \xi}{1-G\left(x_{i} ; \xi\right)}-b \sum_{i=1}^{n} G\left(x_{i} ; \xi\right) \xi\left(1-G\left(x_{i} ; \xi\right)\right)^{-b-1} \\
& +(a-1) \sum_{i=1}^{n} \frac{G\left(x_{i} ; \xi\right) \xi\left(1-G\left(x_{i} ; \xi\right)\right)^{-b-1}}{e^{\left(1-G\left(x_{i} ; \xi\right)\right)^{-b}-1}-1}
\end{aligned}
$$

where $G\left(x_{i} ; \xi\right) \xi$ and $g\left(x_{i} ; \xi\right) \xi$ denote the derivatives of $G\left(x_{i} ; \xi\right)$ and $g\left(x_{i} ; \xi\right)$ with respect to $\xi$, respectively.

For instance, in the framework of the ETIWIW distribution, we have $\xi=(\mu, \theta)$, and so, after some algebraic manipulations, we find

$$
\begin{gathered}
G(x ; \mu, \theta)_{\mu}=-\theta \mu^{\theta-1} x^{-\theta} e^{-(\mu / x)^{\theta}}, \quad g(x ; \mu, \theta)_{\mu}=\theta^{2} \mu^{\theta-1} x^{-2 \theta-1} e^{-(\mu / x)^{\theta}}\left(x^{\theta}-\mu^{\theta}\right), \\
G(x ; \mu, \theta)_{\theta}=-e^{-(\mu / x)^{\theta}}\left(\frac{\mu}{x}\right)^{\theta} \log \left(\frac{\mu}{x}\right)
\end{gathered}
$$

and

$$
g(x ; \mu, \theta)_{\theta}=\mu^{\theta} x^{-\theta-1} e^{-(\mu / x)^{\theta}}\left[\theta \log (\mu)-\theta\left(\frac{\mu}{x}\right)^{\theta} \log \left(\frac{\mu}{x}\right)-\theta \log (x)+1\right] .
$$

The solutions of the non-linear equations: $U_{b}=0, U_{a}=0$ and $U_{\xi}=0$ with respect to $b, a$ and $\xi$ give the maximum likelihood estimates (MLEs) of $(a, b, \xi)$, denoting by $(\hat{a}, \hat{b}, \hat{\xi})$. One can notice that, thanks to second equation, the MLEs are related by the following simple relation:

$$
\hat{a}=-\left\{\frac{1}{n} \sum_{i=1}^{n} \log \left[1-e^{1-\left(1-G\left(x_{i} ; \hat{\xi}\right)\right)^{-\hat{b}}}\right]\right\}^{-1} .
$$


However, as an evidence, there is no closed-form for these estimates. For a given $G(x ; \xi)$, one can approximate them numerically by the use of any mathematical software. Furthermore, the well-known theory on the maximum likelihood method holds. In particular, subject to some conditions of regularity, the multivariate distribution $\mathcal{N}_{d}\left(0, J^{-1}\right)$, where $d$ indicates the total number of parameters and $J$ denotes the observed Fisher information matrix, is useful to determine the estimated squared errors (SEs) of the MLEs, asymptotic confidence intervals and likelihood ratio statistical tests involving the model parameters.

\section{Numerical Studies}

This section is devoted to some inferential features of the ETIWIW model as described in Section 2.3, with applications to three practical data sets. All the computations are carried out by the use of the R software.

\subsection{Simulation Work}

Here, we come up with a simulation work to investigate the behavior of MLEs in the context of the ETIWIW model. In this regard, we generate 5000 random samples of size $n=50,100,200$, 500 and 1000 from the ETIWIW distribution by the inverse transform sampling using the qf given by (6). With the order $(a, b, \mu, \theta)$ in mind, six sets of parameters are assigned as, Set1 $=(0.5,0.5,0.5,0.5)$, Set2 $=(1.5,1.5,0.5,1.5)$, Set3 $=(1.8,1.5,0.5,3)$, Set4 $=(0.8,0.6,0.5,0.8)$, Set5 $=(1.2,0.8,0.5,0.5)$, and Set $6=(1.5,0.8,0.6,1)$. Then, the MLEs of $a, b, \mu$ and $\theta$, as well as their mean squared errors (MSEs) are documented in Tables 2 and 3.

Table 2. MLEs and MSEs of the ETIWIW model parameters for Set1, Set2 and Set3.

\begin{tabular}{|c|c|c|c|c|c|c|}
\hline \multirow{2}{*}{$n$} & \multicolumn{2}{|c|}{ Set1 } & \multicolumn{2}{|c|}{ Set2 } & \multicolumn{2}{|c|}{ Set3 } \\
\hline & MLE & MSE & MLE & MSE & MLE & MSE \\
\hline \multirow{4}{*}{50} & 0.747 & 0.159 & 1.028 & 0.734 & 1.377 & 1.031 \\
\hline & 0.507 & 0.065 & 1.729 & 1.568 & 1.704 & 1.279 \\
\hline & 0.729 & 0.436 & 0.768 & 0.128 & 0.678 & 0.059 \\
\hline & 0.707 & 0.093 & 2.711 & 2.992 & 3.322 & 2.939 \\
\hline \multirow{4}{*}{100} & 0.737 & 0.153 & 1.318 & 0.599 & 1.463 & 0.825 \\
\hline & 0.502 & 0.027 & 1.522 & 0.631 & 1.393 & 0.449 \\
\hline & 0.662 & 0.227 & 0.698 & 0.073 & 0.641 & 0.034 \\
\hline & 0.662 & 0.046 & 2.227 & 0.896 & 3.204 & 2.127 \\
\hline \multirow{4}{*}{200} & 0.718 & 0.124 & 1.31 & 0.29 & 1.6 & 0.501 \\
\hline & 0.487 & 0.014 & 1.241 & 0.361 & 1.229 & 0.268 \\
\hline & 0.66 & 0.22 & 0.636 & 0.036 & 0.605 & 0.019 \\
\hline & 0.662 & 0.036 & 2.204 & 0.827 & 3.075 & 1.481 \\
\hline \multirow{4}{*}{500} & 0.786 & 0.12 & 1.394 & 0.208 & 1.644 & 0.344 \\
\hline & 0.475 & 0.0063 & 1.184 & 0.185 & 1.209 & 0.149 \\
\hline & 0.475 & 0.09 & 0.611 & 0.02 & 0.598 & 0.013 \\
\hline & 0.645 & 0.026 & 2.168 & 0.55 & 2.943 & 1.037 \\
\hline \multirow{4}{*}{1000} & 0.761 & 0.087 & 1.394 & 0.125 & 1.673 & 0.241 \\
\hline & 0.464 & 0.0042 & 1.15 & 0.165 & 1.335 & 0.125 \\
\hline & 0.449 & 0.03 & 0.601 & 0.015 & 0.584 & 0.0084 \\
\hline & 0.651 & 0.025 & 2.152 & 0.499 & 2.948 & 0.975 \\
\hline
\end{tabular}

We see that the MLEs are closed to the true values of parameters. Furthermore, the MSEs consistently decreases when the sample size increases. We conclude that the maximum likelihood method is efficient to estimate the ETIWIW model parameters.

In the three next subsections, we apply the ETIWIW model to three practical data sets having different characteristics, showing how it can outperform some other notable models of the literature. 
Table 3. MLEs and MSEs of the ETIWIW model parameters for Set4, Set5 and Set6.

\begin{tabular}{ccccccc}
\hline \multirow{4}{*}{$n$} & \multicolumn{2}{c}{ Set4 } & \multicolumn{2}{c}{ Set5 } & \multicolumn{2}{c}{ Set6 } \\
\cline { 2 - 7 } & MLE & MSE & MLE & MSE & MLE & MSE \\
\hline \multirow{4}{*}{50} & 1.016 & 0.934 & 1.138 & 0.087 & 1.272 & 1.642 \\
& 1.299 & 2.379 & 0.438 & 0.054 & 1.76 & 3.227 \\
& 1.604 & 4.595 & 0.926 & 0.279 & 1.461 & 2.553 \\
& 1.2 & 0.391 & 0.93 & 0.413 & 1.309 & 0.637 \\
\hline \multirow{4}{*}{100} & 0.968 & 0.48 & 1.152 & 0.06 & 1.304 & 1.152 \\
& 1.034 & 0.574 & 0.445 & 0.03 & 1.359 & 0.719 \\
& 1.146 & 1.367 & 0.927 & 0.244 & 1.097 & 0.919 \\
& 1.05 & 0.175 & 0.813 & 0.173 & 1.183 & 0.29 \\
\hline \multirow{4}{*}{200} & 0.985 & 0.398 & 1.249 & 0.042 & 1.477 & 1.021 \\
& 0.908 & 0.184 & 0.455 & 0.019 & 1.221 & 0.45 \\
& 0.905 & 0.611 & 0.862 & 0.18 & 0.88 & 0.498 \\
& 1.01 & 0.108 & 0.756 & 0.1 & 1.102 & 0.196 \\
\hline \multirow{5}{*}{500} & 0.978 & 0.309 & 1.238 & 0.018 & 1.485 & 0.352 \\
& 0.734 & 0.035 & 0.463 & 0.0075 & 0.994 & 0.136 \\
& 0.65 & 0.119 & 0.852 & 0.162 & 0.672 & 0.092 \\
& 0.937 & 0.074 & 0.713 & 0.054 & 1.096 & 0.131 \\
\hline \multirow{6}{*}{1000} & 0.907 & 0.158 & 1.229 & 0.011 & 1.511 & 0.109 \\
& 0.686 & 0.024 & 0.465 & 0.0061 & 0.985 & 0.103 \\
& 0.57 & 0.033 & 0.815 & 0.13 & 0.617 & 0.046 \\
& 0.854 & 0.065 & 0.711 & 0.053 & 1.049 & 0.093 \\
\hline \multirow{6}{*}{} & & & & & &
\end{tabular}

\subsection{Application to the Rainfall Data Set}

First of all, we consider the "rainfall data set" which consists of the mean of maximum daily rainfall for 30 years (from 1975 to 2004) at 35 stations in the middle and west of peninsular Malaysia. This data set was recently studied by [41]. It contains the following values: 1.134, 1.196, 1.181, 1.178, $1.048,1.077,0.835,1.163,0.880,1.056,1.164,0.914,1.141,1.068,1.007,1.027,1.298,0.842,0.991,0.955$, $0.703,0.953,1.018,1.003,1.106,1.110,1.249,1.092,1.187,1.047,0.989,0.955,1.234,0.937,0.933$.

The data are unimodal, moderately left skewed, with a platykurtic form of the histogram. In view of its shape properties demonstrated in Figures 1 and 2, the ETIWIW model must be able to treat such data.

Then, we aim to compare the fitting performances of the ETIWIW model with those of fairly comparable models, i.e., based on modifications of the Weibull or Fréchet distributions and with three or four parameters. That is, the following notorious ones are considered, with references: the generalized odd gamma Fréchet (GOFr) model by [42], log-gamma generated Fréchet (LGFr) model by [43], Kumaraswamy Fréchet (KwFr) model by [44], beta Fréchet (BFr) model by [45], exponentiated Fréchet (EFr) model by [46] and type II truncated Fréchet inverse Weibull (TIITFIW or equivalently, TIWIW) model by [21].

As a first approach, for all these models, we determine the MLEs and SEs of the parameters. The results are provided in Table 4 .

Then, as statistical benchmarks, the following goodness-of-fit measures are considered: minus complete log-likelihood $(-\hat{\ell})$, Akaike information criterion (AIC), Anderson-Darling $\left(\mathrm{A}^{*}\right)$, Cramer-von Mises ( $\left.\mathrm{W}^{*}\right)$, and Kolmogorov-Smirnov (K-S) statistic. The lower the values of these measures, the better the fit. Furthermore, the $p$-value of the K-S test is provided. A largest $p$-value is always preferable for fitting purposes. The numerical results are collected in Table 5 .

Based on Table 5, the ETIWIW model is the best, with the smallest goodness-of-fit measures, i.e., $-\hat{\ell}(-\hat{\ell}=80.8218)$, AIC (AIC $=169.6438), \mathrm{W}^{*}\left(\mathrm{~W}^{*}=0.0540\right), \mathrm{A}^{*}\left(\mathrm{~A}^{*}=0.4239\right), \mathrm{K}-\mathrm{S}(\mathrm{K}-\mathrm{S}=0.1185)$ and largest $p$-value ( $p$-value $=0.6276)$. We note that the best challenger is the TIITFIW (or TIWIW) 
model, i.e., which corresponds to the ETIWIW model defined with $a=1$, with more that 2 points of difference for the AIC, among others.

All the fits of the estimated pdfs and cdfs are drawn in Figure 3.

Table 4. MLEs and SEs of the models parameters for the rainfall data set.

\begin{tabular}{ccccccc}
\hline Model & $\boldsymbol{a}$ & $\boldsymbol{b}$ & $\boldsymbol{\mu}$ & $\boldsymbol{\theta}$ & $\boldsymbol{\alpha}$ & $\boldsymbol{\beta}$ \\
\hline \multirow{2}{*}{ ETIWIW } & 0.4443 & 477.2912 & 118.7575 & 0.6907 & - & - \\
& $(0.1084)$ & $(8.6456)$ & $(5.4174)$ & $(0.0392)$ & - & - \\
\hline \multirow{2}{*}{ TIITFIW } & - & 40.7759 & 62.1370 & 0.6356 & - & - \\
& - & $(7.9297)$ & $(1.14315)$ & $(0.0982)$ & - & - \\
\hline \multirow{2}{*}{ LGFr } & 0.2832 & 23.0128 & 21.4924 & 1.3365 & - & - \\
& $(0.0413)$ & $(7.8511)$ & $(2.2458)$ & $(1.1915)$ & - & - \\
\hline \multirow{2}{*}{ GOFr } & - & - & 13.0778 & 4.6917 & 0.1739 & 1.1748 \\
& - & - & $(9.1451)$ & $(3.4160)$ & $(0.8502)$ & $(0.3630)$ \\
\hline \multirow{2}{*}{ KwFr } & 5.2239 & 554.6118 & 9.8825 & 0.5019 & - & - \\
& $(36.8518)$ & $(22.0773)$ & $(38.8463)$ & $(0.0927)$ & - & - \\
\hline \multirow{2}{*}{ BFr } & - & - & 71.1502 & 259.1749 & 74.9748 & 0.1685 \\
& - & - & $(12.1141)$ & $(30.5647)$ & $(1.0465)$ & $(3.1490)$ \\
\hline EFr & - & - & 1.6108 & 1.9444 & 7.9293 & - \\
& - & - & $(67.4174)$ & $(1.0673)$ & $(1.0274)$ & - \\
\hline
\end{tabular}

Table 5. Values of $-\hat{\ell}, \mathrm{AIC}, \mathrm{W}^{*}, \mathrm{~A}^{*}, \mathrm{~K}-\mathrm{S}$ and the related $p$-value for the rainfall data set.

\begin{tabular}{ccccccc}
\hline Model & $-\hat{\ell}$ & AIC & $\mathbf{W}^{*}$ & $\mathbf{A}^{*}$ & K-S & $\boldsymbol{p}$-Value \\
\hline ETIWIW & 80.8218 & 169.6438 & 0.0540 & 0.4239 & 0.1185 & 0.6276 \\
TIITFIW & 82.9131 & 171.8263 & 0.0905 & 0.6681 & 0.1274 & 0.5340 \\
LGFr & 88.9168 & 185.8337 & 0.2372 & 1.5496 & 0.1820 & 0.1412 \\
GOFr & 87.8787 & 183.7575 & 0.2201 & 1.4468 & 0.1353 & 0.4562 \\
KwFr & 86.1506 & 180.3014 & 0.1710 & 1.1615 & 0.1218 & 0.5928 \\
BFr & 91.2658 & 190.5317 & 0.3161 & 1.9815 & 0.1523 & 0.3109 \\
EFr & 101.5918 & 209.1836 & 0.6067 & 3.4796 & 0.2438 & 0.0172 \\
\hline
\end{tabular}
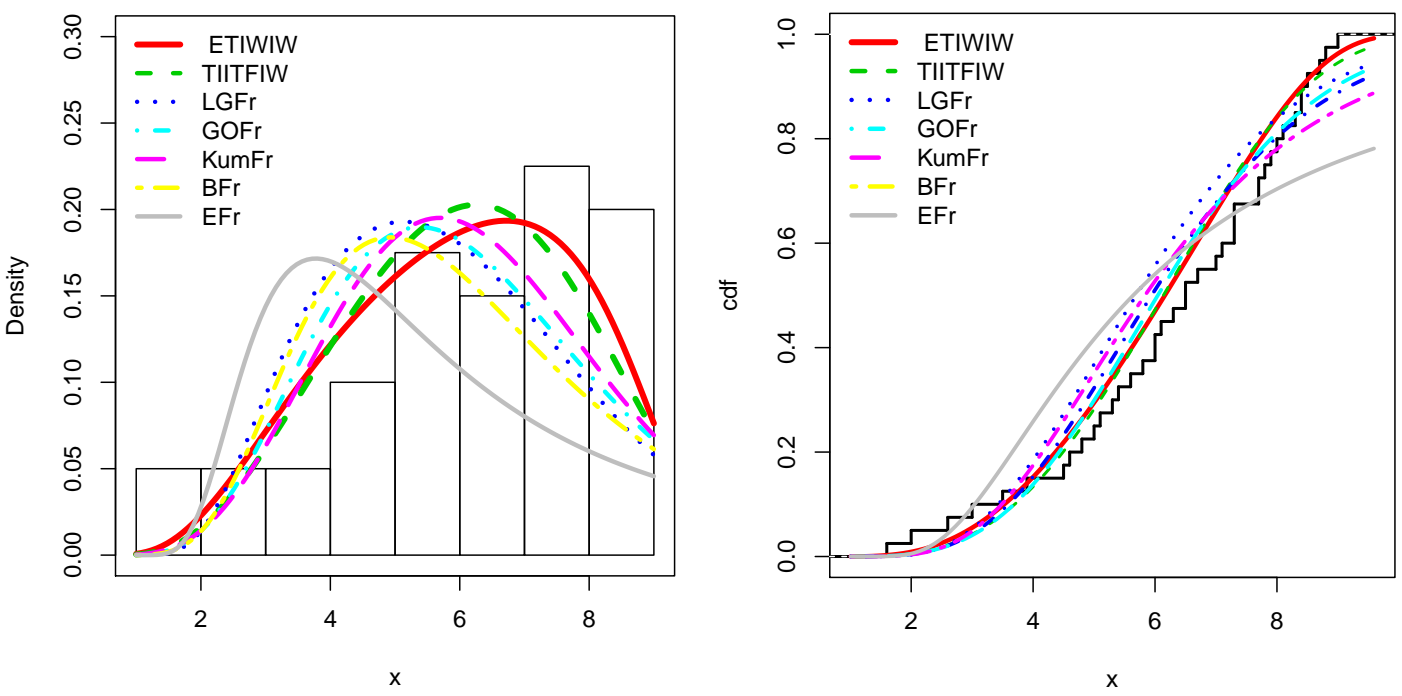

Figure 3. Estimated pdfs and cdfs for rainfall data set.

Figure 3 indicates that the fits of the ETIWIW model seem more adequate in comparison to the concurrence; the round form of the histogram is well captured thanks to the platykurtic nature of the 
ETIWIW model, less pronounced for the TIITFIW (or TIWIW) model. In this sense, the rainfall data set is well adjusted by the ETIWIW model.

\subsection{Application to the Sum of Skin Folds Data Set}

Adopting the same mathematical framework than above, we now apply the ETIWIW model to another data set, with different characteristics, called the "sum of skin folds data set" and coming from [47]. The data set contains sums of skin folds in 202 athletes collected at the Australian Institute of Sports. It contains the following values: $28.0,98,89.0,68.9,69.9,109.0,52.3,52.8,46.7,82.7,42.3$, 109.1, 96.8, 98.3, 103.6, 110.2, 98.1, 57.0, 43.1, 71.1, 29.7, 96.3, 102.8, 80.3, 122.1, 71.3, 200.8, 80.6, 65.3, 78.0, $65.9,38.9,56.5,104.6,74.9,90.4,54.6,131.9,68.3,52.0,40.8,34.3,44.8,105.7,126.4,83.0,106.9,88.2,33.8$, 47.6, 42.7, 41.5, 34.6, 30.9, 100.7, 80.3, 91.0, 156.6, 95.4, 43.5, 61.9, 35.2, 50.9, 31.8, 44.0, 56.8, 75.2, 76.2, $101.1,47.5,46.2,38.2,49.2,49.6,34.5,37.5,75.9,87.2,52.6,126.4,55.6,73.9,43.5,61.8,88.9,31.0,37.6$, $52.8,97.9,111.1,114.0,62.9,36.8,56.8,46.5,48.3,32.6,31.7,47.8,75.1,110.7,70.0,52.5,67,41.6,34.8$, $61.8,31.5,36.6,76.0,65.1,74.7,77.0,62.6,41.1,58.9,60.2,43.0,32.6,48,61.2,171.1,113.5,148.9,49.9$, $59.4,44.5,48.1,61.1,31.0,41.9,75.6,76.8,99.8,80.1,57.9,48.4,41.8,44.5,43.8,33.7,30.9,43.3,117.8,80.3$, 156.6, 109.6, 50.0, 33.7, 54.0, 54.2, 30.3, 52.8, 49.5, 90.2, 109.5, 115.9, 98.5, 54.6, 50.9, 44.7, 41.8, 38.0, 43.2, 70.0, 97.2, 123.6, 181.7, 136.3, 42.3, 40.5, 64.9, 34.1, 55.7, 113.5, 75.7, 99.9, 91.2, 71.6, 103.6, 46.1, 51.2, 43.8, $30.5,37.5,96.9,57.7,125.9,49.0,143.5,102.8,46.3,54.4,58.3,34.0,112.5,49.3,67.2,56.5,47.6,60.4,34.9$.

These data are known to be unimodal, leptokurtic and highly right skewed; as suggested by Figures 1 and 2, the ETIWIW model is appropriate for data having such characteristics.

After computations, the MLEs and SEs of the considered models are collected in Table 6.

Table 6. MLEs and SEs for the sum of skin folds data set.

\begin{tabular}{ccccccc}
\hline Model & $\boldsymbol{a}$ & $\boldsymbol{b}$ & $\boldsymbol{\mu}$ & $\boldsymbol{\theta}$ & $\boldsymbol{\alpha}$ & $\boldsymbol{\beta}$ \\
\hline ETIWIW & 1.5665 & 0.0580 & 28.7074 & 16.2632 & - & - \\
& $(0.1228)$ & $(0.0024)$ & $(0.0290)$ & $(0.0292)$ & - & - \\
\hline \multirow{2}{*}{ TIITFIW } & - & 0.1709 & 34.8247 & 5.9024 & - & - \\
& - & $(0.0755)$ & $(2.5283)$ & $(2.2193)$ & - & - \\
\hline \multirow{2}{*}{ LGFr } & 6.6285 & 2.9542 & 21.9999 & 2.0724 & - & - \\
& $(2.0456)$ & $(2.0521)$ & $(1.2151)$ & $(0.1112)$ & - & - \\
\hline \multirow{2}{*}{ GOFr } & - & - & 4.8711 & 12.9533 & 0.0626 & 0.6005 \\
& - & - & $(2.9411)$ & $(1.1160)$ & $(0.2531)$ & $(0.0680)$ \\
\hline \multirow{2}{*}{ KWFr } & 16.8895 & 486.2870 & 5.3100 & 0.3782 & - & - \\
& $(4.0633)$ & $(2.0475)$ & $(2.1463)$ & $(0.0058)$ & - & - \\
\hline \multirow{2}{*}{ BFr } & - & - & 122.7997 & 230.4647 & 88.7582 & 0.1570 \\
& - & - & $(2.1441)$ & $(30.1642)$ & $(7.0361)$ & $(0.1290)$ \\
\hline \multirow{2}{*}{ EFr } & - & - & 11.0292 & 2.6174 & 53.4457 & - \\
& - & - & $(6.0124)$ & $(0.1432)$ & $(5.0211)$ & - \\
\hline
\end{tabular}

Then, numerical results of the considered criteria are presented in Table 7.

From Table 7, the ETIWIW model reveals to be the best, with the smallest $-\hat{\ell}(-\hat{\ell}=946.3521)$, AIC $($ AIC $=1900.7040), \mathrm{W}^{*}\left(\mathrm{~W}^{*}=0.0838\right), \mathrm{A}^{*}\left(\mathrm{~A}^{*}=0.6006\right), \mathrm{K}-\mathrm{S}(\mathrm{K}-\mathrm{S}=0.0645)$ and largest $p$-value $(p$-value $=0.3646)$.

The fits of the estimated pdfs and cdfs are presented in Figure 4.

Figure 4 illustrates the sharp fits involved by the ETIWIW model. In particular, we see that the mode of the data set is particularly well captured by the estimated pdf of the ETIWIW model, in comparison to the concurrence. 
Table 7. Values of $-\hat{\ell}, \mathrm{AIC}, \mathrm{W}^{*}, \mathrm{~A}^{*}, \mathrm{~K}-\mathrm{S}$ and the related $p$-value for the sum of skin folds data set.

\begin{tabular}{ccccccc}
\hline Model & $-\hat{\ell}$ & AIC & $\mathbf{W}^{*}$ & $\mathbf{A}^{*}$ & K-S & $p$-Value \\
\hline ETIWIW & 946.3521 & 1900.7040 & 0.0838 & 0.6006 & 0.0645 & 0.3646 \\
TIITFIW & 947.5516 & 1901.1030 & 0.0905 & 0.6789 & 0.0663 & 0.3366 \\
LGFr & 953.4254 & 1914.8510 & 0.1722 & 1.1660 & 0.0722 & 0.2419 \\
GOFr & 959.3028 & 1926.6060 & 0.3388 & 1.9968 & 0.0836 & 0.1181 \\
KWFr & 963.5626 & 1935.120 & 0.4373 & 2.5605 & 0.0921 & 0.0645 \\
BFr & 955.9227 & 1919.8450 & 0.2539 & 1.5299 & 0.0714 & 0.2542 \\
EFr & 955.8263 & 1917.6530 & 0.1998 & 1.4661 & 0.0706 & 0.2660 \\
\hline
\end{tabular}
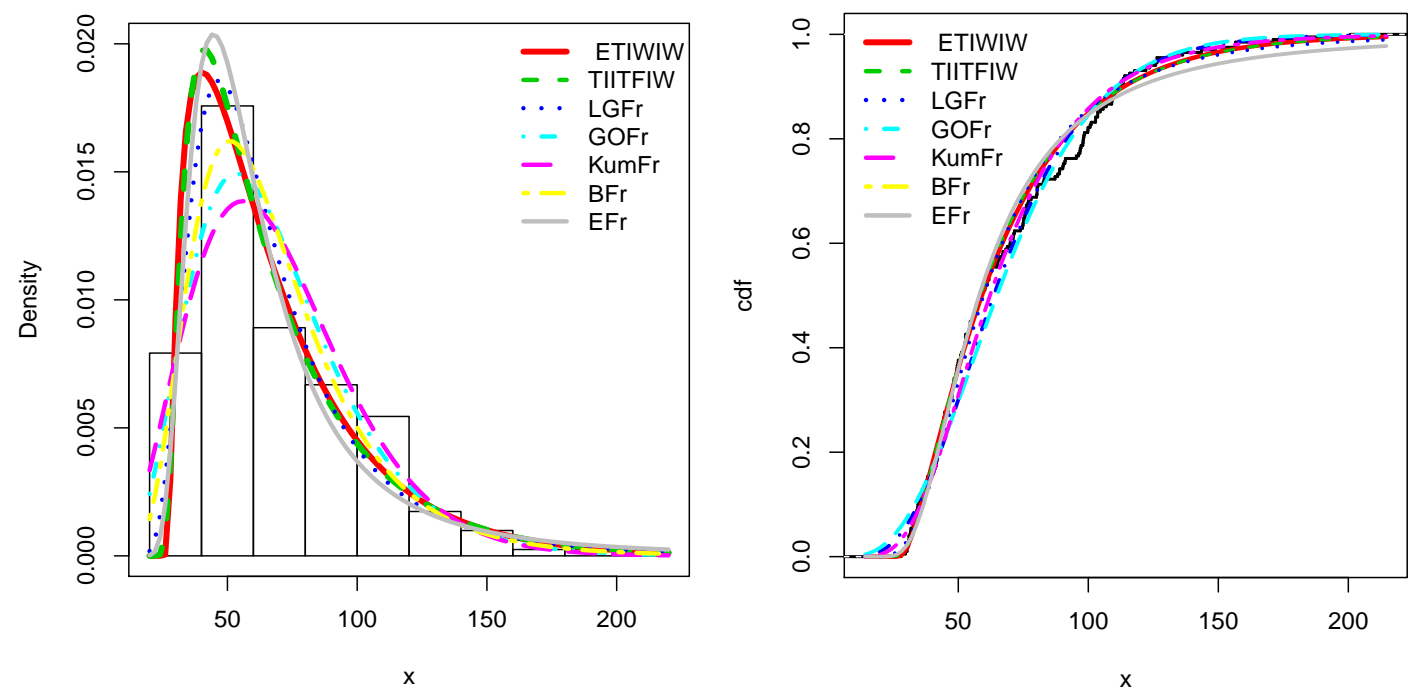

Figure 4. Estimated pdfs and cdfs for the sum of skin folds data set.

\subsection{Application to the Completed Passes of Drew Brees Data Set}

Go on with the same methodology, we now apply the ETIWIW model to a modern sport data set called the "completed passes of Drew Brees data set", reported by the National Centers for Environmental Information available at: http://www.espn.com/nfl/player/gamelog/_/id/2580/ year/2016. The data are 16 sums of completed American football passes performed by Andrew Christopher (Drew) Brees (a famous quarterback) durging the matchs of the 2016 regular season (from Sun $9 / 11$ to Sun 1/1 2016). The data are: 28, 29, 36, 23, 34, 37, 27, 28, 21, 35, 28, 31, 25, 37, 23, 29. A basic histogram of the data shows that they are unimodal, moderately right skewed, and rather moderate platykurtic. Thus, the properties of this data set differ to the previous ones. As shown in Figures 1 and 2, the ETIWIW model is adapted for such data, as developed below.

After computations, Table 8 presents the MLEs and SEs of the considered models.

Then, numerical results of the considered criteria are presented in Table 9.

From Table 9, we conclude that the ETIWIW model is the best, with the smallest $-\hat{\ell}(-\hat{\ell}=48.3270)$, AIC $($ AIC $=104.6542), \mathrm{W}^{*}\left(\mathrm{~W}^{*}=0.0516\right), \mathrm{A}^{*}\left(\mathrm{~A}^{*}=0.3559\right), \mathrm{K}-\mathrm{S}(\mathrm{K}-\mathrm{S}=0.1219)$ and largest $p$-value $(p$-value $=0.9438)$. Furthermore, we note that the TIITFIW (or TIWIW) model is the worst of all in terms of AIC (and in the middle of the list for the other criteria), illustrating the importance of the exponentiated transformation proposed in the ETIWIW model (with power parameter $a$ estimated by $\hat{a}=2.5869$, far from 1 ).

As last approach, the fits of the estimated pdfs and cdfs are plotted in Figure 5.

We see in Figure 5 the fit behavior of the ETIWIW model, capturing all the necessary informations of the data. 
Table 8. MLEs and SEs for the completed passes of Drew Brees data set.

\begin{tabular}{ccccccc}
\hline Model & $\boldsymbol{a}$ & $\boldsymbol{b}$ & $\boldsymbol{\mu}$ & $\boldsymbol{\theta}$ & $\boldsymbol{\alpha}$ & $\boldsymbol{\beta}$ \\
\hline \multirow{2}{*}{ ETIWIW } & 2.5869 & 0.1048 & 19.1715 & 21.3741 & - & - \\
& $(0.16813)$ & $(0.4131)$ & $(4.5844)$ & $(3.7627)$ & - & - \\
\hline \multirow{2}{*}{ TIITFIW } & - & 0.4384 & 24.7133 & 7.0868 & - & - \\
& - & $(0.7951)$ & $(6.0332)$ & $(8.6529)$ & - & - \\
\hline \multirow{2}{*}{ LGFr } & 30.2455 & 58.6674 & 26.2278 & 0.8824 & - & - \\
& $(6.9793)$ & $(5.6592)$ & $(1.4252)$ & $(0.1689)$ & - & - \\
\hline \multirow{2}{*}{ GOFr } & - & - & 11.3884 & 26.4729 & 0.1791 & 1.1221 \\
& - & - & $(0.2455)$ & $(2.6733)$ & $(0.0022)$ & $(0.1192)$ \\
\hline \multirow{2}{*}{ KwFr } & 23.4933 & 47.1455 & 9.9122 & 1.5850 & - & - \\
& $(2.2203)$ & $(1.1471)$ & $(2.1669)$ & $(0.2254)$ & - & - \\
\hline \multirow{2}{*}{ BFr } & - & - & 16.4844 & 92.6404 & 72.7783 & 0.6991 \\
& - & - & $(5.9942)$ & $(23.1349)$ & $(8.9369)$ & $(0.0223)$ \\
\hline EFr & - & - & 18.7691 & 6.0666 & 8.2824 & - \\
& - & - & $(864.5581)$ & $(1.1360)$ & $(4.45652)$ & - \\
\hline
\end{tabular}

Table 9. Values of $-\hat{\ell}, \mathrm{AIC}, \mathrm{W}^{*}, \mathrm{~A}^{*}, \mathrm{~K}-\mathrm{S}$ and the related $p$-value for the completed passes of Drew Brees data set.

\begin{tabular}{ccccccc}
\hline Model & $-\hat{\ell}$ & AIC & $\mathbf{W}^{*}$ & A $^{*}$ & K-S & $p$-Value \\
\hline ETIWIW & 48.3270 & 104.6542 & 0.0516 & 0.3559 & 0.1219 & 0.9438 \\
TIITFIW & 49.6099 & 105.2198 & 0.0532 & 0.3646 & 0.1433 & 0.8974 \\
LGFr & 48.4793 & 104.9587 & 0.0523 & 0.3652 & 0.1337 & 0.9369 \\
GOFr & 48.3591 & 104.7184 & 0.0545 & 0.3694 & 0.1382 & 0.9199 \\
KWFr & 48.3594 & 104.7189 & 0.0559 & 0.3751 & 0.1451 & 0.8891 \\
BFr & 48.4446 & 104.8893 & 0.0524 & 0.3639 & 0.1345 & 0.9341 \\
EFr & 49.4775 & 104.9550 & 0.0753 & 0.5013 & 0.1686 & 0.7533 \\
\hline
\end{tabular}
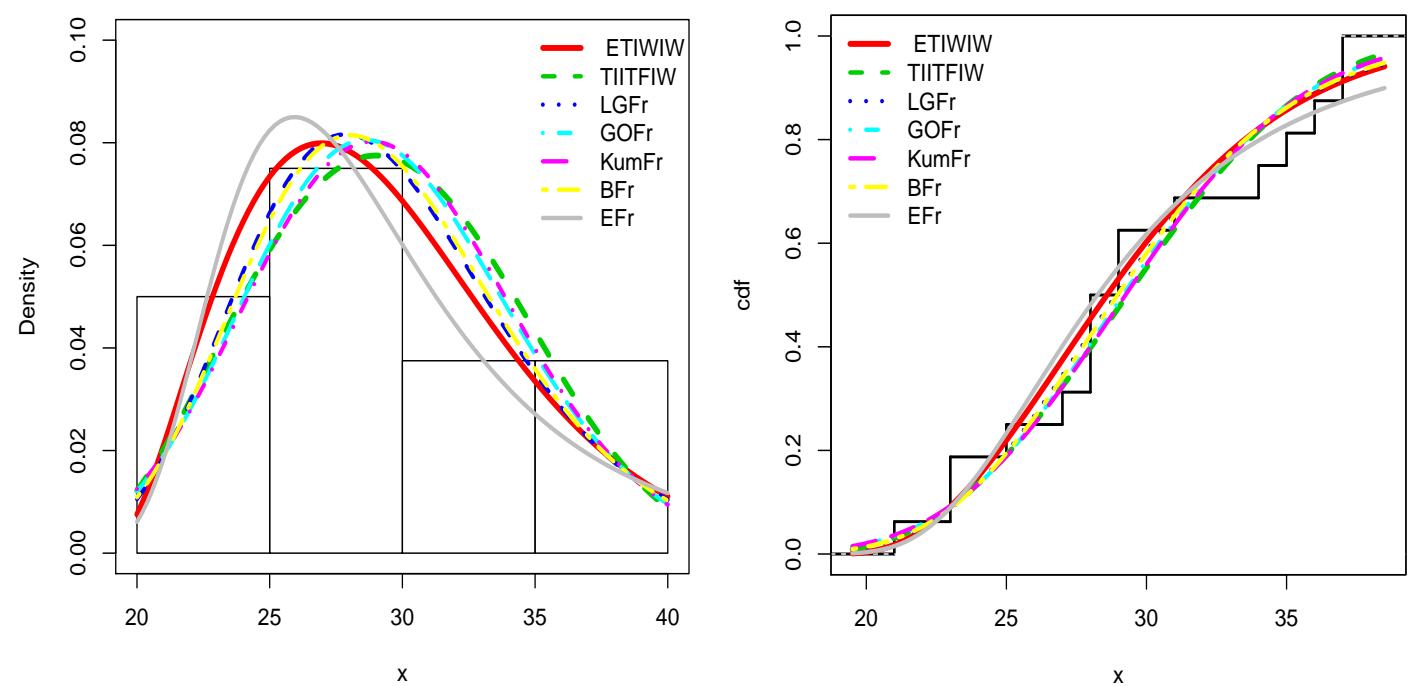

Figure 5. Estimated pdfs and cdfs for the completed passes of Drew Brees data set.

\section{Concluding Remarks and Perspectives}

In this paper, we provide a natural extension of the (TIW-G) family by [21] by the use of the power transform, introducing the exponentiated TIW-G (ETIW-G) family. We have studied its main theoretical and practical features, with a focus on its applicability in a concrete scenario. In particular, by considering the special ETIW-G model defined with the inverse Weibull (IW) model as baseline, 
we shown that it outperforms six competitor models in the fitting of three different practical data sets: the rainfall, sum of skin folds and completed passes of Drew Brees data sets. This study gives the foundation for wider applications in statistics involving the ETIW-G family, believing on its high potential in this regards. One can think to the developments of regression models, as well as the comparison of the performance of the proposed distributions with the existing distributions in this setting.

Author Contributions: A.M.A., M.E., F.J. and C.C. contributed equally to this work. All authors have read and agreed to the published version of the manuscript.

Funding: This work was funded by the Deanship of Scientific Research (DSR), King AbdulAziz University, Jeddah, under grant No. (G: 413-130-1440).

Acknowledgments: We thank the three reviewers for the comments and remarks that have helped to improve this work. This work was supported by the Deanship of Scientific Research (DSR), King AbdulAziz University, Jeddah, under grant No. (G: 413-130-1440). The authors, therefore, gratefully acknowledge the DSR technical and financial support.

Conflicts of Interest: The authors declare no conflict of interest.

\section{References}

1. Gupta, R.D.; Kundu, D. Exponentiated exponential family: An alternative to Gamma and Weibull distributions. Biom. J. 2001, 43, 117-130. [CrossRef]

2. Cordeiro, G.M.; de Castro, M. A new family of generalized distributions. J. Stat. Comput. Simul. 2011, 81, 883-898. [CrossRef]

3. Zografos, K.; Balakrishnan, N. On families of beta- and generalized gamma-generated distributions and associated inference. Stat. Methodol. 2009, 6, 344-362. [CrossRef]

4. Marshall, A.; Olkin, I. A new method for adding a parameter to a family of distributions with applications to the exponential and Weibull families. Biometrika 1997, 84, 641-652. [CrossRef]

5. Shaw, W.T.; Buckley, I.R. The alchemy of probability distributions: Beyond Gram-Charlier expansions, and a skew-kurtotic-normal distribution from a rank transmutation map. arXiv 2009, arXiv:0901.0434.

6. Eugene, N.; Lee, C.; Famoye, F. Beta-normal distribution and its applications. Commun. Stat.-Theory Methods 2002, 31, 497-512. [CrossRef]

7. Torabi, H.; Montazari, N.H. The logistic-uniform distribution and its application. Commun. Stat.-Simul. Comput. 2014, 43, 2551-2569. [CrossRef]

8. Bourguignon, M.; Silva, R.B.; Cordeiro, G.M. The Weibull-G family of probability distributions. J. Data Sci. 2014, 12, 53-68.

9. Brito, C.R.; Rêgo, L.C.; Oliveira, W.R.; Gomes-Silva, F. Method for generating distributions and classes of probability distributions: The univariate case. Hacet. J. Math. Stat. 2019, 48, 897-930.

10. Ahmad, M. On the theory of inversion. Int. J. Stat. Sci. 2007, 6, 43-53.

11. Lehmann, E.L.; Shaffer, J.P. Inverted distributions. Am. Stat. 1988, 42, 833-836.

12. Sheikh, A.K.; Ahmad, M.; Ali, Z. Some remarks on the hazard functions of the inverted distributions. Reliab. Eng. 1987, 19, 255-261. [CrossRef]

13. Hassan, A.; Nassr, G. The inverse Weibull-generator of distributions: Properties and applications. J. Data Sci. 2018, 16, 723-742.

14. Jamal, F.; Chesneau, C.; Elgarhy, M. Type II general inverse exponential family of distributions. J. Stat. Manag. Syst. 2019. [CrossRef]

15. Jamal, F.; Nasir, M.A.; Ozel, G.; Elgarhy, M.; Khan, N.M. Generalized inverted Kumaraswamy generated family of distributions: Theory and applications. J. Appl. Stat. 2019, 46, 2927-2944. [CrossRef]

16. Abid, S.H.; Al-Noor, N.H.; Boshi, M.A.A. On the generalized inverse Weibull distribution. AIP Conf. Proc. 2019, 2086, 030002.

17. Abid, A.H.; Abdulrazak, R.K. [0,1] truncated Fréchet-G generator of distributions. Appl. Math. 2017, 7, 51-66.

18. Jamal, F.; Bakouch, H.S.; Nasir, M.A. A Truncated General-G Class of Distributions with Application to Truncated Burr-G Family. 2019. Available online: https:/ /hal.archives-ouvertes.fr/hal-01956892/document (accessed on 15 March 2020). 
19. Aldahlan, M.A.; Jamal, F.; Chesneau, C.; Elgarhy, M.; Elbatal, I. The truncated Cauchy power family of distributions with inference and applications. Entropy 2019, 22, 346. [CrossRef]

20. Bantan, R.A.R.; Jamal, F.; Chesneau, C.; Elgarhy, M. Truncated inverted Kumaraswamy generated family of distributions with applications. Entropy 2019, 21, 1089. [CrossRef]

21. Aldahlan, M.A. Type II truncated Fréchet generated family of distributions. Int. J. Math. Appl. 2019, 7, 221-228.

22. Murthy, D.P.; Xie, M.; Jiang, R. Weibull Models; John Wiley and Sons: New York, NY, USA, 2004.

23. Lai, C.-D.; Murthy, D.N.; Xie, M. Weibull Distributions and Their Applications; Springer: Berlin, Germany, 2006; pp. 63-78.

24. Shahzad, M.N.; Ullah, E.; Hussanan, A. Beta exponentiated modified Weibull distribution: Properties and application. Symmetry 2019, 11, 781. [CrossRef]

25. de Gusmo, F.R.; Ortega, E.M.; Cordeiro, G.M. The generalized inverse Weibull distribution. Stat. Pap. 2011, 52, 591-619. [CrossRef]

26. Zhang, T.; Xie, M. On the upper truncated Weibull distribution and its reliability implications. Reliab. Eng. Syst. Saf. 2011, 96, 194-200. [CrossRef]

27. Aydin, D. The doubly-truncated exponentiated inverse Weibull distribution. Anadolu Univ. J. Sci. Technol. B-Theor. Sci. 2018, 6, 55-74. [CrossRef]

28. Lehmann, E.L. The power of rank tests. Ann. Math. Stat. 1953, 24, 23-43. [CrossRef]

29. Tahir, M.H.; Cordeiro, G.M.; Alizadeh, M.; Mansoor, M.; Zubair, M.; Hamedani, G.G. The odd generalized exponential family of distributions with applications. J. Stat. Distrib. Appl. 2015, 2, 1-28. [CrossRef]

30. Merovci, F.; Alizadeh, M.; Yousof, H.M.; Hamedani, G.G. The exponentiated transmuted-G family of distributions: Theory and applications. Commun. Stat.-Theory Methods 2017, 46, 10800-10822. [CrossRef]

31. Bantan, R.A.R.; Jamal, F.; Chesneau, C.; Elgarhy, M. Type II Power Topp-Leone Generated Family of Distributions with Statistical Inference and Applications. Symmetry 2020, 12, 75. [CrossRef]

32. Aldahlan, M.A.; Jamal, F.; Chesneau, C.; Elbatal, I.; Elgarhy, M. Exponentiated power generalized Weibull power series family of distributions: Properties, estimation and applications. PLoS ONE 2020, 15, e0230004. [CrossRef]

33. Gómez-Déniz, E.; Iriarte, Y.A.; Calderín-Ojeda, E.; Gómez, H.W. Modified power-symmetric distribution. Symmetry 2019, 11, 1410. [CrossRef]

34. Prasetyo, R.B.; Kuswanto, H.; Iriawan, N.; Ulama, B.S.S. Binomial regression models with a flexible generalized logit link function. Symmetry 2020, 12, 221. [CrossRef]

35. Klein, J.P.; Moeschberger, M.L. Survival Analysis: Techniques for Censored and Truncated Data, 2nd ed.; Springer: Berlin, Germany, 2003.

36. Kenney, J.F.; Keeping, E.S. Mathematics of Statistics, 3rd ed.; Pt. 1; Van Nostrand: Princeton, NJ, USA, 1962.

37. Moors, J.J.A. A quantile alternative for kurtosis. J. R. Stat. Soc. Ser. 1988, 37, 25-32. [CrossRef]

38. Green Wood, J.A.; Landwehr, J.M.; Matalas, N.C.; Walti, J.R. Probability weighted moments; Definition and relation to parameters of distribution expressible in inverse form. Water Resour. Res. 1979, 3, 281-292.

39. Furrer, R.; Naveau, P. Probability weighted moments properties for small samples. Stat. Probab. Lett. 2007, 70, 190-195. [CrossRef]

40. Arnold, B.C.; Balakrishnan, N.; Nagaraja, H.N. A First Course in Order Statistics; John Wiley and Sons: New York, NY, USA, 1992.

41. Khaleel, M.; Ibrahim, N.; Shitan, M.; Merovci, F.; Rehman, E. Beta Burr type-x with application to rainfall data. Malays. J. Math. Sci. 2017, 11, 73-86.

42. Hosseini, B.; Afshari, M.; Alizadeh, M. The generalized odd gamma-G family of distributions: Properties and applications. Austrian J. Stat. 2018, 47, 69-89. [CrossRef]

43. Amini, M.; MirMostafaee, S.M.T.K.; Ahmadi, J. Log-gamma-generated families of distributions. Statistics 2014, 48, 913-932. [CrossRef]

44. Mead, M.E.; Abd-Eltawab, A.R. A note on Kumaraswamy Fréchet distribution. Aust. J. Basic Appl. Sci. 2014, 8, 294-300.

45. Nadarajah, S.; Gupta, A.K. The beta Fréchet distribution. Far East J. Theor. Stat. 2004, 14, 15-24. 
46. Nadarajah, S.; Kotz, S. The Exponentiated Fréchet Distribution, InterStat. Available online: http:/ / interstat. statjournals.net/YEAR/2003/articles/0312002.pdf (accessed on 15 March 2020).

47. Weisberg, S. Applied Linear Regression; John Wiley and Sons: New York, NY, USA, 2005; Volume 528.

(C) 2020 by the authors. Licensee MDPI, Basel, Switzerland. This article is an open access article distributed under the terms and conditions of the Creative Commons Attribution (CC BY) license (http://creativecommons.org/licenses/by/4.0/). 